CAHIERS DE

NARRATOLOGIE

\section{Cahiers de Narratologie}

Analyse et théorie narratives

17 | 2009

Stéréotype et narration littéraire

\title{
Du type au stéréotype : analyse des modalités d'insertion des stéréotypes des physiologies dans Bouvard et Pécuchet
}

\section{Vincent Stohler}

\section{OpenEdition}

Journals

Édition électronique

URL : http://journals.openedition.org/narratologie/1184

DOI : 10.4000/narratologie. 1184

ISSN : 1765-307X

Éditeur

LIRCES

Référence électronique

Vincent Stohler, « Du type au stéréotype : analyse des modalités d'insertion des stéréotypes des physiologies dans Bouvard et Pécuchet », Cahiers de Narratologie [En ligne], 17 | 2009, mis en ligne le 05 janvier 2011, consulté le 19 avril 2019. URL : http://journals.openedition.org/narratologie/1184 ; DOI : 10.4000/narratologie. 1184

Ce document a été généré automatiquement le 19 avril 2019

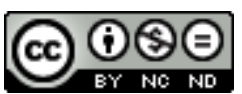

Cahiers de Narratologie - Analyse et théorie narratives est mis à disposition selon les termes de la licence Creative Commons Attribution - Pas d'Utilisation Commerciale - Pas de Modification 4.0 International. 


\title{
Du type au stéréotype : analyse des modalités d'insertion des stéréotypes des physiologies dans Bouvard et Pécuchet
}

\author{
Vincent Stohler
}

«Tous les lieux communs sur les âges, les sexes et

les tempéraments

leur semblèrent de la plus haute importance » Bouvard et Pécuchet, Flaubert ${ }^{1}$

1 Dans le cadre de cette étude, nous tenterons d'analyser la manière dont Flaubert récupère les stéréotypes présents dans les physiologies de son époque, en cherchant à définir leurs différentes modalités d'insertion dans Bouvard et Pécuchet. A la suite des travaux d'Anne Herschberg-Pierrot ${ }^{2}$ et de Laurent Adert $^{3}$ qui se sont intéressés à l'enjeu des stéréotypes existants dans les discours échangés par les personnages diégétiques, nous voudrions montrer que la stéréotypie flaubertienne est également à la source de tout un travail de composition qui influe directement sur la construction intrinsèque du texte : composition des personnages (traits physiques, caractériels, axiologiques, etc.), composition structurelle du roman, etc. Mais avant cela, nous aimerions rapidement faire le point sur la notion de stéréotype ainsi que sur ses différentes variantes casuelles que sont le cliché, le poncif et le lieu commun.

2 Qu'est-ce qu'un stéréotype ? Si la notion a suscité, et suscite encore, un vif intérêt depuis que Walter Lippmann l'a introduite dans son ouvrage Opinion publique et plus largement dans les sciences sociales en $1922^{4}$, elle aura été aussi génératrice de confusion en autorisant une multiplicité de formulations selon le domaine de recherche dans lequel elle est abordée. Dans les études littéraires, le problème se pose également. Le stéréotype est bien souvent assimilé à des termes qui lui sont voisins et qui ne cessent d'en brouiller les contours, certains critiques utilisant indistinctement des vocables comme cliché, poncif 
, lieu commun ou encore idée reçue. Pour y voir plus clair, le plus sûr est de se tourner vers les principaux chercheurs qui se sont penchés dernièrement sur le sujet et de prendre en compte les traits définitoires qu'ils ont retenus : nous pensons tout particulièrement à Ruth Amossy ${ }^{5}$, ainsi qu'à Anne Herschberg-Pierrot ${ }^{6}$, Claude Boucher ${ }^{7}$ et Jean-Louis Dufays ${ }^{8}$

De leurs différentes recherches, il ressort de manière constante que le terme stéréotype désigne au sens large une structure, une association d'éléments qui est susceptible d'affecter tous les niveaux du discours : idées, thèmes, expressions, actions, etc. Mais pour que cette structure puisse être identifiée comme étant une association stéréotypée, elle doit impérativement répondre aux quelques critères suivants :

- Pour assurer sa pérennité, le stéréotype doit être fréquemment répété par le groupe social dans lequel il se définit.

- A force d'être utilisé, le stéréotype s'est figé, l'association de ses termes a acquis une certaine fixité, ce qui lui permet d'être reproduit tel quel (on retrouve ici le sens étymologique des mots cliché, poncif, et stéréotype).

- L'origine du stéréotype n'est pas clairement identifiable, elle est le plus souvent multiple.

- Le stéréotype se donne sous forme de condensé, de schématisation simplificatrice et abstraite qui ne rend que partiellement compte de la complexité de la réalité.

- Le stéréotype s'inscrit dans la mémoire collective d'un groupe social de manière durable.

Pour éviter toute confusion, certains théoriciens ont également proposé une terminologie précise qui permet de classer les stéréotypes en fonction du niveau d'abstraction de leurs composants : cliché, lorsque le stéréotype se situe sur un plan proprement linguistique et qu'il reproduit une structure syntagmatique ou phrastique ${ }^{10}$; poncif, lorsque le stéréotype agit sur un plan thématique ou narratif, reproduisant des thèmes littéraires ${ }^{11}$ (décors, personnages, actions, scénarios, schémas) et lieu commun ou idée reçue ${ }^{12}$, lorsque le stéréotypeagit sur un plan idéologique (représentations mentales, propositions, valeurs ${ }^{13}$ .)Le terme de stéréotype est quant à lui réservé pour qualifier l'ensemble du phénomène.

5 Après cette mise au point théorique, venons-en à Flaubert et plus précisément à son dernier roman qui met en scène un couple, un personnage double. Un choix qui contraste certes avec ses autres textes, mais qui se justifie pleinement : deux personnages, constate Claude Digeon, « c'est la possibilité permanente de dialogue, de contraste de caractère, de discussion d'idées ${ }^{14} »$. Créés pour parler, Bouvard et Pécuchet le feront dès leur rencontre et jusqu'à la dernière page du roman, immergeant le lecteur dans un flot incessant de paroles ${ }^{15}$. Mais cette parole acquiert dans le texte un statut particulier. Bien souvent, les deux personnages, s'ils parlent de tout, parlent aussi pour ne riendire, se faisant l'écho d'un déjà-dit. Leurs discours, se greffant sur la rumeur publique, ne se construisent qu'à partir d'une parole antérieure, commune ${ }^{16}$. Anne Herschberg-Pierrot dans son étude Le dictionnaire des idées reçues ${ }^{17}$, ainsi que Laurent Adert dans Les mots des autres ${ }^{18}$ ont clairement montré comment Flaubert a su, dans un premier temps, capter les lieux communs de son époque, les classer selon leurs thèmes et en proposer un dictionnaire (Le fameux Dictionnaire des idées reçues qui constitue la seconde partie de Bouvard et Pécuchet), puis, dans un second temps, les exploiter dans le cadre de sa fiction en faisant en sorte qu'ils alimentent les discours de ses personnages. Par exemple, comme le constate Laurent Adert, «si, à propos de quelques visites d'églises, le roman dit que Bouvard et Pécuchet 'déploraient le vandalisme, tonnaient contre le badigeon ${ }^{19}$ ', nous lisons dans le Dictionnaire[des idées reçues], au mot badigeon: 'Dans les églises. Tonner contre. Cette colère artistique est bien portée ${ }^{20}$; si, en visite au Musée d'histoire naturelle, 'ce qu'ils 
admirèrent du cèdre, c'est qu'on l'eût rapporté dans un chapeau ${ }^{21}$, l'article cèdre du Dictionnaire indique que celui du Jardin des Plantes 'a été rapporté dans un chapeau ${ }^{22}$ '.$^{23}$ Le lieu commun devient ainsi le médiateur des discours des personnages, constituant à la fois son origine et sa substance.

Mais nous pensons qu'il n'y a pas que les paroles des personnages qui se trouvent investies de stéréotypes dans le roman flaubertien, et que ceux-ci se propagent à l'ensemble du récit selon différentes modalités d'insertion. Pour poursuivre notre analyse dans cette direction, nous aimerions nous intéresser plus précisément au cas des "physiologies", genre littéraire en vogue dans la première moitié du XIXe siècle, car nous pensons qu'elles ont une grande incidence dans le travail de stéréotypie de Flaubert tel qu'il est à l'œuvre dans Bouvard et Pécuchet. Tentons, tout d'abord, de définir de quoi il s'agit.

7 Le Grand Larousse Universel du XIXe siècle donne la définition suivante du mot " physiologie »: «Des études de mœurs décorées du nom de physiologie ont été assez longtemps à la mode; vers 1840, on se mit à faire des physiologies, comme au XVIIe siècle on faisait des portraits, et toutes les professions, tous les types caractéristiques furent analysés dans leurs moindres particularités. On eut la physiologie de l'avocat et la physiologie du portier, celle du notaire et celle de la marchande de quatre saisons. [...] Le domaine de la physiologie ainsi comprise est vaste ; il englobe absolument tout ${ }^{24}$. $\gg$ Les auteurs de physiologies se donnent donc pour mission de décrire la société de leur temps en étudiant les mœurs de leurs contemporains. Leur but, similaire à celui des rédacteurs des grands dictionnaires qui voient le jour plus ou moins au même moment, est de catégoriser le réel pour le rendre plus intelligible, c'est-à-dire de subdiviser la population en milieux, professions, conditions sociales, etc. A la « rage » taxinomique du projet (le XIXème siècle est l'âge d'or du "classement ») fait pendant une volonté de monstration, d'instruction du public sur la société dans laquelle il vit. Jules Janin, dans la préface de sa grande encyclopédie de mœurs (probablement la plus célèbre de son temps) Les Français peints par eux-mêmes, dresse clairement le projet de son entreprise :

Chaque année a ses mœurs et ses caractères qui lui sont propres; [...]les historiens, oubliant l'espèce humaine, se sont amusés à raconter des sièges, des batailles, des villes prises et renversées, des traités de paix ou de guerre, toutes sortes de choses menteuses, sanglantes et futiles; ils ont dit comment se battaient les hommes et non pas comment ils vivaient ; ils ont décrit avec le plus grand soin leurs armures, sans s'inquiéter de leur manteau de chaque jour; ils se sont occupés des lois, non pas des mœurs. [...] Pourquoi donc n'aurions-nous pas, nous aussi le peuple frivole et mobile par excellence, un registre tout exprès pour $\mathrm{y}$ transcrire ces nuances si fines, si déliées, et pourtant si vraies, de nos mœurs chaque jour? [...] Il est nécessaire que cette longue tâche de l'étude des mœurs se divise et se subdivise à l'infini, que chacune de ces régions lointaines choisisse un historien dans son propre lieu, que chacun parle de ce qu'il a vu et entendu dans le pays qu'il habite ${ }^{25}$.

8 La méthode paraît simple : les « historiens » qui participent au projet se voient attribuer un objet particulier d'analyse dont ils doivent dresser un modèle représentatif (un type) en en réunissant les caractéristiques essentielles. Le but est de joindre le singulier à l'universel et d'offrir une représentation typique dans laquelle toute une catégorie humaine se définit. Si le projet est noble - expliquer le réel dans un esprit de simplification et de recoupement -, il en résulte que les différents types qui constituent les encyclopédies de mœurs se voient dans la pratique construits pour beaucoup autour des préjugés ou des fantasmes qui s'égrènent dans les collectivités. En d'autres termes, les physiologies reproduisent et propagent des schèmes figés sans que leurs auteurs en aient 
véritablement conscience ${ }^{26}$. « De fait, [constate Ruth Amossy] la première moitié du XIXe siècle manifeste une réelle incapacité à penser le concept de stéréotype face à celui, sacrosaint, de type ${ }^{27}$. Et lorsque les physiologies se voient critiquées par les élites intellectuelles de leur temps, ce n'est que pour des raisons de style bâclé ou parce qu'elles constituent une œuvre alimentaire pour celui qui la produit, mais jamais à cause du savoir qu'elles délivrent et de son rapport au réel ${ }^{28}$. Au contraire, les images des catégories sociales qu'elles diffusent réussissent à s'imposer. Walter Benjamin dans une étude sur Baudelaire ${ }^{29}$ a montré que si le type des physiologies connaît un grand succès dans une France postrévolutionnaire en mal de repères, c'est qu'il porte en lui un caractère rassurant, qu'il transforme le mystère inquiétant de la foule des villes en un inventaire bien agencé, procurant aux gens une représentation aimable de tout un chacun. Car dans les physiologies, l'Autre est toujours perçu sous les apparences d'une bonhommie inoffensive.

9 A partir du XXe siècle, cette position s'inverse radicalement. Les types sont dès lors considérés comme une simplification, une manière d'enfermer le réel dans un moule préconçu ; en d'autres termes, une façon de promouvoir une représentation trompeuse de la complexité de la réalité, de la diversité des classes sociales et plus généralement des individus ${ }^{30}$. Flaubert, en précurseur, entend déconstruire les stéréotypes présents dans les physiologies en les réexploitant dans ses romans,mais en leur imprimant un recul critique et en adoptant à leur égard un positionnement idéologico-discursif « indécidable» au sens derridien du terme ${ }^{31}$. Si la démarche s'apparente à ce que nous avons pu déjà observer lorsque nous évoquions les paroles de Bouvard et Pécuchet et leurs liens au Dictionnaire des idées reçues ${ }^{32}$, ici la réappropriation des schèmes figés va permettre un déploiement accru de leurs modalités d'insertion dans le récit. Tentons de saisir, par exemple, le travail de récupération qu'effectue Flaubert à partir des différentes physiologies du bourgeois, probablement l'un des types qui ont été le plus largement diffusés dans les encyclopédies de mœurs, et mesurons l'impact de ces physiologies au niveau de la construction narrative du roman.

Tout d'abord, on constate qu'elles interfèrent directement dans le travail de composition des personnages. Dès les premières pages du récit flaubertien, le ton est donné: l'euphorie de la rencontre de Bouvard et Pécuchet sur le canal Saint-Martin consiste dans la reconnaissance jubilatoire de leurs ressemblances. Ils s'arrêtent à la même minute, s'asseyent sur le même banc, retirent tous les deux en même temps leurs couvre-chefs où sont inscrits de la même manière leurs noms, se découvrent la même profession, partagent les mêmes opinions, et ainsi de suite ${ }^{33}$. D'emblée, le caractère schématique, récurrent et figé des deux portraits ne peut manquer de sauter aux yeux du lecteur. Mais en affinant l'analyse, on se rend compte que la composition des traits essentiels des deux personnages (traits physiques, moraux, caractériels, etc.) n'est pas le fruit du hasard ou l'expression d'une individualité qui leur serait propre, mais découle d'un code de construction qui façonne un modèle cohérent, faisant lui-même directement écho à celui des physiologies.

11 Considérons par exemple leur apparence. En recoupant différentes sources, il ressort de manière constante que le type du bourgeois est un homme qui " a passé la quarantaine ${ }^{34}$ " et dont les goûts vestimentaires se résument à quelques objets qui permettent de l'identifier. Toujours couvert, il porte un « chapeau ${ }^{35}$ », sa « redingote $^{36}$ » est de couleur sombre, le plus souvent «brune ${ }^{37}$ »et dissimule immanquablement un « gilet $^{38}$ » dont il se sépare que très rarement par peur de prendre froid; il est également chaussé de 
"souliers en castor ${ }^{39}$ ", lesquels sont recouverts de «larges pantalons ${ }^{40}$ »; sa " cravate [est] de mousseline blanche ${ }^{41}$ ». Et «presque tous les individus sont armés de cannes et d'une tabatière d'où ils tirent une poudre noire avec laquelle ils farcissent incessamment leur nez $\mathrm{z}^{42} »$. Autant d'éléments qui se trouvent subtilement intégrés dans les premières descriptions de Bouvard et Pécuchet :

Deux hommes parurent. L'un venait de la Bastille, l'autre du Jardin des Plantes. Le plus grand, vêtu de toile, marchait le chapeau en arrière, le gilet déboutonné et sa cravate à la main. Le plus petit, dont le corps disparaissait dans une redingote marron, baissait la tête sous une casquette à visière pointue. [...] L'aspect aimable de Bouvard charma de suite Pécuchet. Ses yeux bleuâtres, toujours entreclos, souriaient dans son visage colore. Un pantalon à grand-pont, qui godait par le bas sur des souliers de castor, moulait son ventre, faisait bouffer sa chemise à la ceinture ${ }^{43}$. trouve façonné selon les impératifs du modèle préfabriqué disponible dans les encyclopédies de mours et se réapproprie l'ensemble de ses traits constitutifs (chapeau, redingote, souliers de castor, etc.). En passant de la physiologie à son doublet romanesque, on change certes de registre, mais dans les deux cas on retrouve la même association d'éléments qui renvoie au même schème représentationnel figé. Le type s'inversant en stéréotype, ce sont donc les lieux communs puisés par Flaubert dans les physiologies qui gouvernent dans Bouvard et Pécuchet l'apparence des personnages, et qui en permettent la représentation. Sauf que l'accumulation artificielle dans le récit flaubertien de tous ces éléments porte en elle un geste critique dans la mesure où elle confère à Bouvard et Pécuchet une apparence improbable, ce qui ne peut, d'une part, passer inaperçu aux yeux du lecteur et, surtout, ce qui l'engage à reconsidérer le rapport simplificateur et fallacieux qu'entretiennent les lieux communs avec le réel. Il en résulte que les physiologies, alors qu'elles se donnaient pour but de construire un modèle cognitif, empirique - c'était du moins le projet des encyclopédistes - se trouvent dès lors remises en question, contestées.

15 A considérer le tempérament de Bouvard et Pécuchet, on remarque que la stratégie compositionnelle des personnages s'apparente à ce que nous venons de décrire. Leurs manières de penser, leurs opinions, ou plus largement leurs goûts ne leur sont pas spécifiques, mais sont directement comparables à ceux que partage l'ensemble du groupe dont ils sont issus. L'évocation dans le récit de leurs occupations de prédilection est à ce titre révélateur. Selon les physiologies, le bourgeois aime à regarder tout ce qui l'entoure, va observer « les nouveautés du Museum ${ }^{46}$ », les « expositions de tableaux ${ }^{47}$ »; il apprécie également "les promenades, les concerts, les spectacles ${ }^{48}$ »; il se passionne pour l'avancement des travaux publics de sa ville ${ }^{49}$, « examine par lui-même le dallage de la place Louis $\mathrm{XV}^{50}$ "; et «ce qu'il souhaite le plus : une séance de l'Académie française ${ }^{51}$ ", etc. Bref, « tout est fête, étonnement, matière à examen pour lui ${ }^{2}$ ! » Pas étonnant que pour sceller leur nouvelle amitié, Bouvard et Pécuchet tombent tout de suite d'accord sur le choix de leurs premières occupations : 
Ils flânaient le long des boutiques de bric-à-brac. Ils visitèrent le Conservatoire des Arts et Métiers, Saint-Denis, les Gobelins, les Invalides, et toutes les collections publiques. [...] Dans les galeries du Muséum, ils passèrent avec ébahissement devant les quadrupèdes empaillés, avec plaisir devant les papillons. [...] Ils s'efforcèrent au Louvre de s'enthousiasmer pour Raphaël. A la grande bibliothèque ils auraient voulu connaître le nombre exact des volumes. Une fois, ils entrèrent au cours d'arabe du Collège de France ; et le professeur fut étonné de voir ces deux inconnus qui tâchaient de prendre des notes. Grâce à Barberou, ils pénétrèrent dans les coulisses d'un petit théâtre. Dumouchel leur procura des billets pour une séance de l'Académie ${ }^{53}$.

Et lorsque Bouvard se trouve seul :

sa distraction était, le dimanche, d'inspecter les travaux publics ${ }^{54}$.

Flaubert n'a ainsi pas cherché à développer pour chacun de ses personnages une personnalité qui lui serait propre; au contraire, il a fait tout l'inverse en constituant Bouvard et Pécuchet comme une représentation fidèle de la psyché du bourgeois, ou du moins de l'idée que l'on s'en fait à son époque. Le schème abstrait du stéréotype est repérable ici, non pas dans les paroles ou l'apparence des personnages comme c'était le cas auparavant, mais dans les valeurs (ce qu'ils aiment, ce qu'ils n'aiment pas, leurs raisonnements, etc.) qui sous-tendent leurs choix et leurs agissements. Les lieux communs des physiologies s'investissent ainsi dans le récit de manière détournée. Remarquons encore l'usage comique du trope présuppositionnel ${ }^{55}$ («Ils s'efforcèrent au Louvre de s'enthousiasmer pour Raphaël $\left.{ }^{56} »\right)$, où le verbe «s'efforcer » laisse apparaître à la fois le caractère prescriptif du lieu commun sous-jacent au roman («le bourgeois aime la peinture de Raphaël») et l'incapacité de Bouvard et Pécuchet à correspondre spontanément à cette norme.

Pour terminer, intéressons-nous à la composition structurelle du roman, car elle suscite un certain nombre de remarques. On le sait, à la base de l'écriture de son dernier texte réside pour Flaubert l'ambition d'un vieux projet: se moquer de tous les savoirs, démontrer leurs limites face au réel et prendre ainsi à contre-pied l'esprit scientiste de son temps. Ceci permet de mieux saisir le statut particulier de Bouvard et Pécuchet que son auteur définissait lui-même comme " une encyclopédie critique en farce ${ }^{57}$ ». Si l'ambition $\mathrm{du}$ projet semblait claire dès le départ, sa réalisation et surtout sa forme ne l'étaient pas pour autant : « il me faut un semblant d'action, une espèce d'histoire continue pour que la chose n'ait pas l'air d'une dissertation philosophique ${ }^{58}$. $)$ Au final, Flaubert imagine une histoire qui nous raconte les péripéties de deux commis qui, lassés de leur travail, ont décidé de se retirer à la campagne pour y réaliser leur vieux rêve : se remettre à l'étude, se cultiver, apprendre une multitude de savoirs. Une trame narrative aussi minimale qu'efficace, car ainsi définie elle permet d'y intégrer in extenso une multiplicité d'épisodes en lien avec les différentes sciences que l'auteur entendait aborder. Mais cette simplicité structurelle cache en son fond une construction qui répond à un code particulier : aussi minimaux soient-ils, les événements qui rythment les premiers chapitres du texte se basent sur un schéma de construction qui leur préexiste. A y regarder de plus près, on constate que le roman se façonne à partir de la trame narrative d'un autre texte, celui d'une physiologie. En effet, en 1842 paraît le type du Bourgeois campagnard ${ }^{59}$ écrit par Frédéric Soulié et publié dans l'encyclopédie de mœurs Les Français peints par eux-mêmes. Dans cette étude, l'auteur tente de décrire, sur la base de ce qui se dit sur le sujet ${ }^{60}$, le destin commun des bourgeois, qui ont fait le choix de quitter la capitale pour s'établir à la campagne, en une succession d'événements obligés auxquels ils ont dû faire face. Quoi 
qu'il en soit, il en ressort que l'étude est un ramassis de lieux communs. Or c'est bien sa structure événementielle qui va guider la construction de la trame narrative de Bouvard et Pécuchet. Pour en donner la preuve, et pour mesurer à quel point Flaubert entendait lier le destin de ses deux personnages à celui, stéréotypé, du «bourgeois campagnard ", nous avons comparé la composition structurelle des deux textes sous la forme du tableau récapitulatif ci-dessous. La colonne de gauche renvoie aux séquences narratives du Bourgeois campagnard, la colonne de droite à celles de Bouvard et Pécuchet.

\begin{tabular}{|c|c|c|}
\hline & $\begin{array}{l}\text { Structure narrative du Bourgeois campagnard } \\
61\end{array}$ & Structure narrative de Bouvard et Pécuchet \\
\hline a) & $\begin{array}{l}\text { L'étude de Soulié s'ouvre par un démenti: } \\
\text { "On s'imagine en général que le bourgeois de } \\
\text { Paris est citadin, qu'il a l'amour de sa ville. } \\
\text { On se trompe, [...] car de tous les Français le } \\
\text { bourgeois de Paris est le plus } \\
\text { champêtre. »(p.25) «Boutiquier ou commis, } \\
\text { enchainé derrière un comptoir ou en face } \\
\text { d'un bureau »(p.25), ses journées se } \\
\text { ressemblent toutes. }\end{array}$ & $\begin{array}{l}\text { Bouvard et Pécuchet commence par la } \\
\text { rencontre des deux commis sur le canal } \\
\text { Saint-Martin; identiques à tous points, } \\
\text { comme nous l'avons vu, les deux } \\
\text { personnages partagent à la fois la même } \\
\text { profession, mais surtout le même ennui, «la } \\
\text { monotonie du bureau » (p.62) Ils se sentent } \\
\text { pareillement «fatigué[s] de la capitale.» } \\
(\text { p.53) }\end{array}$ \\
\hline b) & $\begin{array}{l}\text { Le bourgeois se projette dans un avenir } \\
\text { meilleur: «la campagne est le rêve de toutes } \\
\text { ses heures, [...] il lui emprunte le courage } \\
\text { nécessaire à supporter la dure épreuve de la } \\
\text { vie citadine.» (p.25); « Ne viendra-t-il pas un } \\
\text { jour où il ira cueillir lui-même sa tendre } \\
\text { laitue et ses légumes croquants une heure } \\
\text { avant de se mettre à table?»(p.26) }\end{array}$ & $\begin{array}{l}\text { Pour supporter leur quotidien, les deux } \\
\text { compères nourrissent en secret un rêve } \\
\text { commun, se retirer à la campagne, «posséd } \\
\text { [er] un domicile à eux, [où] ils mangeraient } \\
\text { les poules de leur basse-cour, les légumes de } \\
\text { leur jardin »(p.66) C'est d'ailleurs l'une de } \\
\text { leurs premières exclamations : «Comme on } \\
\text { serait bien à la campagne!»(p.52) }\end{array}$ \\
\hline c) & $\begin{array}{l}\text { D'ici-là, c'est un rituel, «le dimanche venu, } \\
\text { nos citadins se précipitent hors de la cité " } \\
\text { (p.25), bravent le soleil, la poussière, se } \\
\text { couchent dans l'herbe au pied d'un arbre, y } \\
\text { mangent. }\end{array}$ & $\begin{array}{l}\text { N'ayant pas les moyens de leurs ambitions, } \\
\text { le dimanche, ils sortent de Paris, } \\
\text { "vagabond[ent] entre les vignes, arach[ent] } \\
\text { des coquelicots au bord des champs, dorm } \\
\text { [ent] sur l'herbe, [...], mang[ent] sous les } \\
\text { acacias [...] et rentr[ent] fort tard, } \\
\text { poudreux, exténués, ravis.»(p.62) }\end{array}$ \\
\hline d) & $\begin{array}{l}\text { Et finalement, lorsque « le marchand a vendu } \\
\text { son fonds, le commis a obtenu sa retraite, ils } \\
\text { ont à leur disposition un capital. » (p.28) Le } \\
\text { rêve peut enfin se réaliser. }\end{array}$ & $\begin{array}{l}\text { Et puis, un jour, le miracle se produit, } \\
\text { Bouvard hérite comme par magie. La } \\
\text { décision est prise : ils réaliseront leur rêve. } \\
\text { "Mais comme [Pécuchet] ne voulait point } \\
\text { vivre aux crochets de Bouvard, il ne } \\
\text { partirait pas avant sa retraite. (pp. 65-66) }\end{array}$ \\
\hline
\end{tabular}


e) en vaines recherches jusqu'à ce qu'il tombe dans les mains d'un homme d'affaires qui [lui propose] une maison de campagne. » (p.28)

Seules "quelques réparations aux murs crevassés " (p.28) étaient à prévoir " et la propriété doublera de production!»(p.28); « Et d'abord, il y a à la campagne mille choses qui ne coûtent rien » (p.26). "Le marché se conclut, le bourgeois est propriétaire, il s'installe. » (p.28)

"Souscripteur à la Maison Rustique ou au Dictionnaire d'agriculture " (p.24), le bourgeois ne se lance pas tête baissée dans l'exploitation de son jardin.

h) qu'étiolé, maladif, indigeste» (p.32) ; «[le bourgeois] n'a que les rebuts, les fruits pourris, les légumes secs, les salades montées en graine. » (p.31)

Alors, « le froid arrive, on s'enferme dans la maison [...] Les pluies viennent, [...]on s'ennuie.» (p.31). Le bourgeois " espère le printemps, ce printemps qui répare tout, rajeunit tout, ranime tout, le printemps qui fera reverdir ses semences et son espérance. » (p.31)

j) Mais «son petit capital de roulement se diminue petit à petit sans que tout ce qu'il récolte lui procure une sensible économie. Alors il essaie de se rendre compte de sa dépense, il établit un tableau par doit et avoir.» (p.30) Le constat est amer, le bourgeois doit «se déterminer à vendre sa maison » (p.32)

k) De retour à Paris, le bourgeois ambitionne de reprendre son ancienne profession.
Vient le difficile choix de la région où ils iront s'établir, trouver une maison à acquérir. "Après dix-huit mois de recherches, ils n'avaient rien trouvé.» (p.67) C'est finalement Barberou, un ami, qui les aide: "on lui avait parlé d'un domaine à Chavignolles, [...] une ferme de trente-huit hectares. »(p.68)

Seul inconvénient, " tous les bâtiments [...] avaient besoin de réparations. » (p.75) Peu importe, ils feraient des économies ailleurs, d'autant que le jardin était prometteur, et Pécuchet "apercevait dans l'avenir des montagnes de fruits, des débordements de fleurs, des avalanches de légumes. »(p.77)

Le déménagement effectué, ils se lancent dans l'agriculture. Pour se garantir un maximum de chance, ils se font conseiller, consultent « la Maison Rustique, [...] s'abonn [ent] à un journal d'agriculture. »(p.84)

Les premières difficultés émergent: "il ne poussa que des végétations rachitiques, [...] Le vent s'amusait à jeter bas les rames des haricots. L'abondance de la gadoue nuisit aux fraisiers, le défaut de pinçage aux tomates. »(p.86)

«Puis les mauvais jours survinrent, [...] ils s'installèrent dans la cuisine, [...] causaient au coin du feu, regardaient la pluie tomber. Dès la mi-carême, ils guettèrent le printemps, et répétaient chaque matin: « tout part. » "(p.78)

A ces premiers ratages s'ajoutent des problèmes financiers. Pécuchet «pendant huit jours travailla les registres [et] découvrit la vérité. [...] Encore deux ans d'une agronomie pareille, leur fortune y passait! Le seul remède était de vendre. »(p.94)

Quand tout leur a craqué entre les mains, par un retour à l'origine, ils reviennent à leurs travaux initiaux. 
On se rend vite compte que les correspondances entre les deux récits sont manifestes. Non seulement les schèmes figés de la physiologie fournissent les thèmes qui seront développés dans les séquences narratives de Bouvard et Pécuchet, mais en plus, elles en instituent la chronologie. La suite d'événements ainsi vécue par les deux commis n'a absolument rien d'original et ne renvoie pas à une réalité qui leur serait propre, mais répond à un code de construction qui s'est façonné à partir de la représentation stéréotypée que se font les gens de la vie du «bourgeois campagnard». Ainsi perçu, le stéréotype devient le véritable « moteur » de la narration flaubertienne.

Pour conclure, nous aimerions citer Flaubert lui-même qui, au moment de démarrer le projet de son dernier texte, déclarait : « il faudrait que dans tout le cours du livre, il n'y eût pas un mot de mon cru et qu'une fois qu'on l'aurait lu, on n'osât plus parler, de peur de dire naturellement une des phrases qui s'y trouvent ${ }^{62}$. » Pari gagné, assurément ! Notre analyse a attiré l'attention sur ce qui paraît être l'une des lignes de force de l'esthétique de l'écrivain : l'amenuisement au sein de l'espace romanesque du langage de l'auteur au profit d'un langage stéréotypé, venu d'ailleurs. Ce phénomène littéraire, s'il n'a de cesse de se développer à partir de Madame Bovary,trouve probablement son ultime aboutissement avec Bouvard et Pécuchet. Ici, comme nous l'avons vu, la stéréotypie flaubertienne a atteint un degré de complexité qui lui permet de toucher toutes les strates de composition du récit romanesque. Flaubert, estimant "qu'il ne faut pas s'écrire ", a donc décidé de se taire, de ne rien dire en son nom propre pour entrer dans une autre relation au langage : écrire n'est plus parler, mais représenter les discours des autres en leur imprimant seulement une mise en scène signifiante, critique. Notre analyse aura donné ainsi preuve aux propos d'Albert Thibaudet qui relevait déjà en 1934 la conception purement citationnelle de l'écriture du dernier ouvrage de Flaubert : "A la limite de Madame Bovary, il y a un livre où il n'y aurait plus besoin de rien mettre en italique, parce que tout devrait y être. C'est Bouvard et Pécuchet. ${ }^{63}$ "

\section{BIBLIOGRAPHIE}

Bouvard et Pécuchet, édition de Claudine Gothot-Mersch, Gallimard, 1979.

Le Dictionnaire des idées reçues, in Bouvard et Pécuchet, Gustave Flaubert, Edition de Claudine

Gothot-Mersch, Gallimard, 1979.

Une leçon d'histoire naturelle. Genre commis, in Les Euvres de jeunesse inédites, Gustave Flaubert, tome I, Louis Conard, éditeur, Paris, 1902.

Les Français peints par eux-mêmes : encyclopédie morale du dix-neuvième siècle, T. 1, L Curmer éditeur, Paris, 1840-1842.

Le bourgeois de Paris, Anaïs Bazin, in Paris ou le livre des cent-et-un, Tome premier, Ladvocat, 1831.

La monographie du rentier in Les Français peints par eux-mêmes : encyclopédie morale du dix-neuvième siècle, T. 3, L Curmer éditeur, Paris, 1840-1842. 
Le Bourgeois campagnard in Les Français peints par eux-mêmes : Encyclopédie morale du dix-neuvième siècle, T. 3, L. Curmer éditeur, Paris, 1840-1842.

L'employé in Les Français peints par eux-mêmes : encyclopédie morale du dix-neuvième siècle, T.1, L Curmer éditeur, Paris, 1840-1842

Correspondance 1871-1877, Gustave Flaubert, Club de l'Honnête Homme éditeur, Paris, 1975.

Correspondance, $3^{\mathrm{ème}} \& 7^{\mathrm{ème}}$ series, Gustave Flaubert, Editions Louis Conard, Librairie éditeur, Paris, 1902.

L'Ere du soupçon, Nathalie Sarraute, Paris, Gallimard, 1956.

Études critiques :

ADERT, LAURENT, Les mots des autres, Flaubert, Sarraute, Pinget, Presses Universitaires du Septentrion, 1996.

AMOSSY, RUTH, Les idées reçues. Sémiologie du stéréotype, Editions Nathan, 1991.

ID., Stéréotypes et clichés, langue, discours, société, Editions Nathan, 1997.

BACKHTINE, MIKHAїL, Le Marxisme et la philosophie du langage, Paris, Minuit, 1977.

Benjamin, WALter, Charles Baudelaire, Paris, Payot, 1982.

BARTHES, ROLAND, S/Z, Paris, Le Seuil, 1970.

BOUCHER, CLAUDE, Lautréamont. Du lieu commun à la parodie, Paris, Larousse, 1974.

DERRIDA, JACQUES, La Dissémination, Paris, Seuil, 1972.

DIGEON, CLAUDE, Le dernier visage de Flaubert, Aubier-Montaigne, Paris, 1946.

DUFAYS,JEAN-LOUIS, Stéréotypie et lecture. Essai sur la réception littéraire, Pierre Mardaga, 1994.

GRIZE, JEAN-BLAISE, Logique naturelle et communications, Paris, PUF, 1996.

HERSCHBERG-PIERROT, ANNE, Problématique du cliché. Sur Flaubert, in « Poétique », nº 43, 1980.

ID., Le Dictionnaire des idées reçues, Presses Universitaires de Lille, 1988.

KERBRAT-ORECCHIONI, CATHERINE, L'Implicite, Paris, Armand Colin, 1986.

THIBAUDET, ALBERT, Gustave Flaubert, Paris, Gallimard, 1963.

\section{NOTES}

1. Gustave Flaubert, Bouvard et Pécuchet, édition de Claudine Gothot-Mersch, Gallimard, 1979, p. 122

2. Anne Herschberg-Pierrot, Le Dictionnaire des idées reçues, Presses Universitaires de Lille, 1988

3. Laurent Adert, Les mots des autres, Flaubert, Sarraute, Pinget, Presses Universitaires du Septentrion, 1996

4. Le terme stéréotype existait auparavant dans le vocabulaire courant dès la fin du XIXe siècle, mais il était utilisé exclusivement dans le domaine de l'imprimerie pour désigner un objet « imprimé avec des planches dont les caractères ne sont pas mobiles, et que l'on conserve pour de nouveaux tirages » (Cf Le Grand Dictionnaire Universel du XIXe siècle)

5. Ruth Amossy, Les idées reçues. Sémiologie du stéréotype, Editions Nathan, 1991 ; Stéréotypes et clichés, langue, discours, société, Editions Nathan, 1997 
6. Anne Herschberg-Pierrot, Problématique du cliché. Sur Flaubert, in Poétique, n³, 1980, p.334-345

7. Claude Boucher, Lautréamont. Du lieu commun à la parodie, Paris, Larousse, "Thème et texte ", 1974

8. Jean-Louis Dufays, Stéréotypie et lecture, Pierre Mardaga, éditeur, 1994

9. «La relation intertextuelle [du stéréotype] opère par prélèvement de passages, d'idées, de schèmes issus de textes antérieurs, et par leur transfert mécanique dans un autre texte auquel ils s'intègrent plus ou moins aisément. [...] Par définition, le stéréotype recouvre plusieurs textes à la fois, dont il est comme une sorte de condensé ou de précipité, en même temps qu'il est schématisation et sclérose. On dira alors que la stéréotypie se réfère à un contexte, c'est-à-dire à la fois ensemble anonyme de texte et ensemble de valeurs véhiculées par ces textes " in Claude Boucher, Lautréamont. Du lieu commun à la parodie, Paris, Larousse, "Thème et texte ", 1974, pp.44-45

10. Selon Ruth Amossy, «le poncif est limité au thème purement littéraire ou poétique, le cliché désigne un fait de style ou une figure de rhétorique usée, le lieu commun se réfère à une opinion partagée et couramment énoncée par le vulgaire. Ainsi le thème du réveil printanier de la nature est un poncif, l'expression « le printemps de la vie » un cliché, et l'idée que le printemps est la saison des amours un lieu commun ou une idée reçue. » Cf Ruth Amossy, op. cit., p.33

11. Ibid.

12. Selon Ruth Amossy, «le lieu commun dans son acception moderne se rapproche de l'idée reçue, expression en vogue dans la seconde moitié du XIXe siècle et au succès de laquelle Le Dictionnaire des idées reçues de Flaubert n'a pas peu contribué aux yeux de la postérité. Rien ne différencie vraiment ces deux termes. »Cf. Ruth Amossy, op. cit., pp.31-32

13. Jean-Louys Dufays qui a également adopté cette distinction tripartite a montré que la distinction en cinq types de structures sémantiques effectuée par Umberto Eco dans son ouvrage Lector in fabula (discursives, narratives, de mondes, actantielles et idéologiques) pouvait s'y subordonner. Cf. Jean-Louys Dufays, Stéréotypie et lecture. Essai sur la réception littéraire, Pierre Mardaga éditeur, Liège, 1994, pp.77-78

14. Claude Digeon, Le dernier visage de Flaubert, Aubier-Montaigne, Paris, 1946, p.142

15. «Leurs paroles coulaient intarissablement, les remarques succédant aux anecdotes, les aperçus philosophiques aux considérations universelles. Ils dénigrèrent le corps des Ponts et chaussées, la régie des tabacs, le commerce, les théâtres, notre marine et tout le genre humain, comme des gens qui ont subi de grands déboires. " in Bouvard et Pécuchet, édition de Claudine Gothot-Mersch, Gallimard, 1979, p. 54

16. A ce sujet, Laurent Adert dans son ouvrage Les mots des autres rapproche la conception bakhtinienne du roman aux textes flaubertiens en tant que «roman explicitement polyphonique » : «L'empire du discours de l'Autre, dont nous constatons la présence au cœur de l'esthétique flaubertienne, marque en tout cas que le sujet - et le romancier tout particulièrement - n'a pas affaire à la langue comme système abstrait de signes mais comme système concret de discours. Ce n'est pas vers Saussure, mais plutôt vers Bakhtine qu'il faut ici se tourner pour saisir cette dimension: 'La langue comme système stable de formes n'est qu'une abstraction savante, qui ne peut servir que des buts théoriques et pratiques particuliers. Cette abstraction ne rend pas compte de façon adéquate de la réalité concrète de la langue. [...] Celle-ci constitue un processus d'évolution ininterrompu, qui se réalise à travers l'interaction verbale sociale des locuteurs. [...] La structure de l'énonciation est une structure purement sociale. Le fait de parole individuel (au sens étroit du mot individuel) est une contradictio in adjecto.' C'est avec une langue de part en part déjà prononcée et accentuée que travaille le romancier[...]. » Laurent Adert, Les mots des autres, Flaubert, Sarraute, Pinget, Presses Universitaires du Septentrion, 1996, pp 52-53 ; (références à Mikhaill Backhtine Cf. Le Marxisme et la philosophie du langage, Paris, Minuit, 1977, p.141)

17. Anne Herschberg-Pierrot, Le Dictionnaire des idées reçues, Presses Universitaires de Lille, 1988 
18. Laurent Adert, Les mots des autres, Flaubert, Sarraute, Pinget, Presses Universitaires du Septentrion, 1996

19. Bouvard et Pécuchet, Gustave Flaubert, Edition de Claudine Gothot-Mersch, Gallimard, 1979, pp.165-166

20. Le Dictionnaire des idées reçues, in Bouvard et Pécuchet, Gustave Flaubert, Edition de Claudine Gothot-Mersch, Gallimard, 1979, p.491

21. Bouvard et Pécuchet, Gustave Flaubert, Edition de Claudine Gothot-Mersch, Gallimard, 1979, p.61

22. Le Dictionnaire des idées reçues, in Bouvard et Pécuchet, Gustave Flaubert, Edition de Claudine Gothot-Mersch, Gallimard, 1979, p.496

23. Laurent Adert, Les mots des autres, Flaubert, Sarraute, Pinget, Presses Universitaires du Septentrion, 1996, p.109

24. Grand dictionnaire universel du XIXe siècle : français, historique, géographique, mythologique, bibliographique...., Pierre Larousse, Paris, 1866-1877,T. 12, p.918

25. Cf. l'introduction de l'ouvrage Les Français peints par eux-mêmes : encyclopédie morale du dixneuvième siècle, T. 1, L Curmer éditeur, Paris, 1840-1842,

26. En empruntant une distinction proposée par Jean-Blaise Grize, on peut considérer que les physiologies prétendent ériger des «modèles» reposant sur «des concepts vrais », mais n'aboutissent qu'à des «schématisations", c'est-à-dire des représentations partielles et orientées, « à la fois tributaire[s] de la finalité du schématisateur et des attentes qu'il prête à son auditoire » (Cf. Jean-Blaise Grize, Logique naturelle et communications, Paris, PUF, coll. « Psychologie sociale ", 1996, p.50)

27. Cf Ruth Amossy, Les idées reçues. Sémiologie du stéréotype, Editions Nathan, 1991 ; Stéréotypes et clichés, langue, discours, société, Editions Nathan, 1997, p.58

28. Comme le constate Ruth Amossy, «le besoin de connaissance et d'ordonnance du social exclut la possibilité de penser la stéréotypie sur le plan de la représentation. » Cf. Ruth Amossy, Les idées reçues. Sémiologie du stéréotype, Editions Nathan, 1991; Stéréotypes et clichés, langue, discours, société, Editions Nathan, 1997, p.58

29. Walter Benjamin, Charles Baudelaire, Paris, Payot, 1982

30. Nathalie Sarraute, pour exemple, dans L'Ere du soupçon, projette de façonner un univers romanesque qui échappe à toute représentation stéréotypée, à toute soumission à un préconstruit, à un déjà pensé ou à un déjà dit, mais qui au contraire adhère le plus possible à la complexité de la réalité : "Ce qui maintenant importe c'est, bien plutôt que d'allonger indéfiniment la liste des types littéraires, de montrer la coexistence de sentiments contradictoires et de rendre, dans la mesure du possible, la richesse et la complexité de la vie psychologique. » in Nathalie Sarraute, L'Ere du soupçon, Paris, Gallimard, « Idées », 1956, p.86

31. Cf. Jacques Derrida, La Dissémination, Paris, Seuil, coll. «Points Essais », 1972, p 156, 272 et passim.

32. La méthode de Flaubert pour dénoncer la prégnance des stéréotypes dans les sociétés sera la même que nous avons pu déjà observer lorsque nous évoquions les discours de Bouvard et Pécuchet : il ne s'agit pas pour lui de les proscrire de ses récits, comme ce sera plus tard le cas avec les avant-gardes, mais au contraire de les reproduire massivement jusqu'à en saturer son texte pour qu'ils se disqualifient d'eux-mêmes, seul moyen selon Roland Barthes pour combattre les schèmes figés : «Le seul pouvoir de l'écrivain sur le vertige stéréotypique (ce vertige qui est aussi celui de la «bêtise », de la "vulgarité »), c'est d'y entrer sans guillemets, en opérant un texte, non une parodie. C'est ce qu'a fait Flaubert dans Bouvard et Pécuchet. » in Roland Barthes, S/ Z, Paris, Le Seuil, 1970, p.104

33. Bouvard et Pécuchet, Gustave Flaubert, Edition de Claudine Gothot-Mersch, Gallimard, 1979, pp.51-52 
34. Le bourgeois de Paris, Anaïs Bazin, in Paris ou le livre des cent-et-un, Tome premier, Ladvocat, 1831, p.23

35. La monographie du rentier in Les Français peints par eux-mêmes : encyclopédie morale du dixneuvième siècle, T. 3, L Curmer éditeur, Paris, 1840-1842, p.1; ainsi que Une leçon d'histoire naturelle. Genre commis, in Les Euvres de jeunesse inédites, Gustave Flaubert, tome I, Louis Conard, éditeur, Paris, 1902, p.199.Il est à noter que le type du «rentier» ou encore celui du «commis » sont considérés par leurs auteurs comme une «subdivision" du type du bourgeois. D'autre part, Flaubert a été lui-même l'auteur d'une physiologie qui lui a été commandée par Le Colibri et qui a été publiée dans ce journal parmi la cinquantaine d'autres en 1837.

36. Ibid.

37. Ibid.

38. Ibid.

39. Une leçon d'histoire naturelle. Genre commis, in Les Euvres de jeunesse inédites, Gustave Flaubert, tome I, Louis Conard, éditeur, Paris, 1902, p.199.

40. Le bourgeois de Paris, Anaïs Bazin, in Paris ou le livre des cent-et-un, Tome premier, Ladvocat, 1831, p.23

41. La monographie du rentier in Les Français peints par eux-mêmes : encyclopédie morale du dixneuvième siècle, T. 3, L Curmer éditeur, Paris, 1840-1842, p.1

42. Ibid.

43. Bouvard et Pécuchet, Gustave Flaubert, Edition de Claudine Gothot-Mersch, Gallimard, 1979, pp.51-52

44. Ibid. pp.57-58

45. Ibid. p.162

46. La monographie du rentier in Les Français peints par eux-mêmes : encyclopédie morale du dixneuvième siècle, T. 3, L Curmer éditeur, Paris, 1840-1842, pp.8-9

47. Ibid., pp.8-9

48. L'employé in Les Français peints par eux-mêmes : encyclopédie morale du dix-neuvième siècle, T. 1, L Curmer éditeur, Paris, 1840-1842, p.302. A noter que le type de « l'employé » est considéré par les auteurs des encyclopédies de mœurs comme une subdivision du type du « bourgeois ».

49. Une leçon d'histoire naturelle. Genre commis, in Les CEuvres de jeunesse inédites, Gustave Flaubert, tome I, Louis Conard, éditeur, Paris, 1902, p.201

50. La monographie du rentier in Les Français peints par eux-mêmes : encyclopédie morale du dixneuvième siècle, T. 3, L Curmer éditeur, Paris, 1840-1842, p.8

51. Ibid., p.9

52. Ibid., pp.8-9

53. Bouvard et Pécuchet, Gustave Flaubert, Edition de Claudine Gothot-Mersch, Gallimard, 1979, pp.60-61

54. Ibid., p.59

55. Catherine Kerbrat-Orecchioni, L'Implicite, Paris, Armand Colin, coll. « U », 1986, p.116

56. Ibid., pp.60-61

57. «C'est l'histoire de ces deux bonshommes qui copient une espèce d'encyclopédie critique en farce. Vous devez en avoir une idée. Pour cela, il va me falloir étudier beaucoup de choses que j'ignore : la chimie, la médecine, l'agriculture. Je suis maintenant dans la médecine. » Lettre envoyée par Flaubert à Mme Roger de Genettes, le samedi 19 août 1872 in Correspondance 1871-1877, Gustave Flaubert, Club de l'Honnête Homme éditeur, Paris, 1975, tome 15, p.149

58. Lettre du $1^{\mathrm{er}}$ avril 1875, A Madame Roger Des Genettes, in Correspondance, Gustave Flaubert, Editions Louis Conard, Librairie éditeur, Paris, 1902, $7^{\mathrm{ème}}$ série, pp.236-238

59. Le Bourgeois campagnard in Les Français peints par eux-mêmes : Encyclopédie morale du dixneuvième siècle, T. 3, L. Curmer éditeur, Paris, 1840-1842 
60. Cf. la note de l'éditeur qui nous apprend que Soulié aurait consulté plus de deux cents personnes avant d'écrire son étude : «M. Soulié avait joint à cet article une suite de plus de deux cents noms avec les adresses mais comme ce recueil repousse tout ce qui ressemble à une personnalité, nous avons cru de notre devoir de supprimer cette liste. (Note de l'éditeur) » in Le bourgeois campagnard in Les Français peints par eux-mêmes : Encyclopédie morale du dix-neuvième siècle, T. 3, L Curmer éditeur, Paris, 1840-1842, p.25

61. La numérotation des pages dans le tableau renvoie à l'édition du Bourgeois campagnard in Les Français peints par eux-mêmes T. 3, L. Curmer éditeur, Paris, 1840-1842, ainsi qu'à l'édition de Claudine Gothot-Mersch, Gallimard, 1979, pour Bouvard et Pécuchet

62. Lettre envoyée à Louise Colet le 17 décembre 1852 in CEuvres complètes de Gustave Flaubert, Correspondance, Troisième série, 1852-1854, Gustave Flaubert, Editions Louis Conard, Paris, 1902, p.67

63. Albert Thibaudet, Gustave Flaubert, Paris, Gallimard, 1963, p.204

\section{RÉSUMÉS}

Cette analyse a pour but d'exposer la manière dont Flaubert récupère les stéréotypes présents dans les physiologies, genre littéraire à la mode dans la première moitié du XIXe siècle, en définissant leurs différentes modalités d'insertion dans Bouvard et Pécuchet. Après s'être interrogée sur le statut particulier des physiologies, l'étude montre que les différents types qu'elles mettent en scène, et plus particulièrement celui du «bourgeois », fournissent à Flaubert un matériel discursif stéréotypé qui lui sert de base de composition à l'ensemble de son texte : composition des personnages (traits physiques, caractériels, axiologiques, etc.), composition structurelle du roman, etc. La démonstration attire ainsi l'attention sur ce qui parait être l'une des lignes de force de l'écriture flaubertienne : l'amenuisement au sein de l'espace romanesque du langage de l'auteur au profit d'un langage stéréotypé, venu d'ailleurs.

\section{INDEX}

Mots-clés : Gustave Flaubert, stéréotype, Bouvard et Pécuchet, physiologie, intertextualité, bourgeoisie, réécriture, narration, personnage, structure

\section{AUTEUR}

\section{VINCENT STOHLER}

Assistant de littérature française moderne à l'Université de Genève 